\title{
Laddering Wave in Serpentine Delay Line
}

\author{
Ruey-Beei Wu \\ Department of Electrical Engineering \\ National Taiwan University, Taipei, Taiwan, R.O.C. \\ Tel: 886-2-3635251 ext. 523, FAX: 886-2-3638247 \\ Fang-Lin Chao \\ Electronic Research Services and Organization \\ Industrial Technology and Research Institute, Hsin-Chu, Taiwan, R.O.C.
}

\begin{abstract}
A wave tracing analysis is proposed to depict the crosstalk-induced laddering wave at receiving end of the serpentine delay line. Quantitative analysis, transient simulation, and measurement are performed to investigate the phenomenon in more details.
\end{abstract}

$0-7803-2411-0 / 94 / \$ 4.00\left(C_{1994}\right.$ IEEE 


\author{
Laddering Wave in Serpentine Delay Line \\ Ruey-Beei $\mathrm{Wu}^{*}$ and Fang-Lin Chao** \\ * Department of Electrical Engineering, National Taiwan University, Taipei \\ Tel: 886-2-3635251 ext. 523, FAX: 886-2-3638247 \\ ** ERSO/Industrial Technology and Research Institute, Hsin-Chu \\ Taiwan, Republic of China
}

\title{
I. INTRODUCTION
}

Synchronization is usually a critical concern in the high speed digital electronic systems. Delay lines are encountered in the design of signal lines to reduce the clock skew of the whole system. A typical example is the serpentine delay line which consists of an odd number of transmission line sections closely packed to each other. Intuitively, the total time delay will increase proportionally to the total length of the delay line. However, the crosstalk noises among these closely packed sections at various time frames may accumulate to cause a drastic deterioration of the total time delay and should be taken into consideration [1].

\section{Wave Tracing Analysis}

Fig.1 shows a nine-section serpentine delay line which is connected to matched resistances at both ends. The driving source $V_{S}(t)$ is a ramped pulse of unit voltage and risetime $t_{\tau}$ which is much smaller than the round-trip time $2 t_{d}$ of a transmission line section. The nine sections are equally spaced to minimize the overall coupling. Let $k_{p}$ denote the average of the capacitive and inductive coupling coefficients between two sections which are separated by $p$ lines.

When the signal launches at the sending end at $t=0$, it will induce backward propagating crosstalk at the near ends of lines \#2, \#4, \#6, and \#8. Since the main signal is of voltage $1 / 2$, these crosstalk noises are of pulse width $2 t_{d}$ and voltages $k_{1} / 4$, $k_{3} / 4, k_{5} / 4$, and $k_{7} / 4$, respectively [2]. As the main signal propagates along line $\# 1$, the crosstalk noises propagate along lines \#3, \#5, \#7, and \#9. They arrive at the far ends at the same time $t=t_{d}$. The main signal then propagates along line \#2 and will induce additional back propagating crosstalk of voltages $k_{1} / 4, k_{3} / 4, k_{5} / 4$, and $k_{7} / 4$ at the far ends of lines $\# 3, \# 5, \# \bar{T}$, and $\# 9$, respectively. As a result, the receiving waveform at the far end of line $\# 9$ will be a crosstalk noise of voltage $k_{7} / 2$ which spans from $t=t_{d}$ to $t=3 t_{d}$.

The induced crosstalk noises, now of voltages $k_{1} / 2, k_{3} / 2$, and $k_{5} / 2$ at far ends of lines \#3, \#5, and \#7, respectively, propagate back along lines \#4, \#6, and \#8, while the main signal propogates along line \#2. These noises will add with the newly induced crosstalk noises when the the main signal reaches the near end of line \#2 and then propagates along line \#3. The coupling and accumulation process repeats until the main signal reaches the far end of line \#9 at $t=9 t_{d}$. Fig.2 shows the resultant waveform at the receiving end. The laddering wave before the arrival of the main signal may become as large as $2 k_{1}$, eight times the value of the near end crosstalk between two transmission lines! If the value $2 k_{1}$ is larger than $1 / 2$, the actual time delay of the nine-section delay line will be $7 t_{d}$ instead of $9 t_{d}$.

III. Quantitative Analysis

The wave tracing analysis assumes a main signal of fixed voltage and neglects the multiple coupling due to the induced crosstalk noises. To accurately predict the magnitude of the laddering wave, a more detailed quantitative analysis is inevitable. 
The voltage waves propagating along coupled transmission lines $\bar{V}(z, t)$ can be written into the superposition of both the forward and backward propagating components, i.e., $\bar{V}^{+}(z, t)$ and $\bar{V}^{-}(z, t)$, respectively. At the near end $z=0$, the backward component $\bar{V}-(0, t)$ is available from that of the far end voltages at a previous time $t-t_{d}$. The forward component $\bar{V}^{+}(0, t)$ can be obtained from the circuit equation which includes the source resistance and the line connection at the near end.

Similarly, the forward component at the far end $\bar{V}^{+}(\ell, t)$ is available from the previously solved $\bar{V}^{+}\left(0, t-t_{d}\right)$. The other component $\bar{V}^{-}(\ell, t)$ can be obtained from the circuit equation at the far end.

Given the cross section of the serpentine delay line shown in Fig.1, the capacitance and inductance matrices $[L]$ and $[C]$ can be calculated $[3]$. The eigen-value and eigenmatrix of $[L] \cdot[C]$ are obtained. The per-unit-length delay of the normal modes varies from 6.69 to $6.99 \mathrm{nsec} / \mathrm{m}$. The average coupling coefficients are about $k_{1}=0.241, k_{2}=$ $0.080, k_{3}=0.033, k_{4}=0.016, k_{5}=0.009, k_{6}=0.006, k_{7}=0.004, k_{8}=0.003$.

Assume that the driver and load resistances are $50 \Omega$. Based on the quantitative analysis, the magnitude of the laddering wave in the receiving end can be obtained. The laddering wave jumps per $2 t_{d}$ and has ladder heights of $0.0038,0.0333,0.1785$, $0.4875,0.5944,0.3650$, and 0.5270 volt in the period $t=t_{d}$ to $15 t_{d}$. It is found that the ladder heights are larger than those predicted by the wave tracing analysis in the light of the multiple coupling effects.

IV. Simulation, Measurement, and Comparisons

The quantitative analysis neglects the effects of the finite risetime and ignores the slight difference among the modal propagation velocities. To investigate the received waveform in more details, the tranient simulation is resorted to.

The wave propagation along the coupled transmission lines can be decomposed into the superposition of the normal modes. The modal voltages are related to the line voltages by a voltage transformation matrix $\left[M_{V}\right]$ which is the eigen-matrix of $[L] \cdot[C]$. The normal modes are decoupled such that each one can be modeled by an isolated transmission line. The transformation from modal voltages to line voltages can be implemented by voltage controlled voltage sources and current controlled current sources at both the near and far ends. Consequently, the nine coupled transmission line sections can be modeled as a SPICE compatible subcircuit [4].

A transient simulation can be performed via the SPICE by defining the line connections and including the exterior circuits at the near and far ends. The risetime of the source is chosen as $t_{r}=350$ psec. Fig. 3 shows the simulated waveforms at the sending and receiving ends in the cases of section length $\ell=5$ and $10 \mathrm{~cm}$. The laddering wave at the receiving end behaves just as predicted by the wave tracing analysis except small ripples near $t=(2 n+1) t_{d l}$ where $n$ is an integer, which is the consequence of the different modal propagation velocities. The saturated heights of all the ladders are in excellent agreement with those by the quantitative analysis.

A testing structure is manufactured and measured by Tek CSA-803. Fig.4 shows the measured data of the received waveform in the cases of $\ell=3,5$, and $10 \mathrm{~cm}$. Although the discrepancy between the theoretical and experimental results is noticeable, the occurrence of the laddering wave in the serpentine delay line is verified clearly.

\section{REFERENCES}

[1] F. L. Chao, "Timing skew of the equal-length serpentines routing," IEEE ASIC Symp., pp. 546-549, 1993. 
[2] A. Feller, H. R. Kaupp, and J. J. Digiacomo, "Crosstalk and reflections in highspeed digital systems," AFIPS Conf. Proc., 1965 Fall Joint Computer Conf., vol. 27, pp. 511-525, Washington D.C.: Spartan Books, 1965.

[3] W. T. Weeks, "Calculation of coefficients of capacitance of multiconductor transmission lines in the presence of a dielectric interface," IEEE Trans. Microwave Theory Tech, vol. 18, pp. 35-43, Jan. 1970.

[4] F. Romeo and M. Santomaro, "Time-domain simulation of n coupled transmission lines," IEEE Trans. Microwave Theory Tech., vol. 35, pp. 131-136, Feb. 1987.

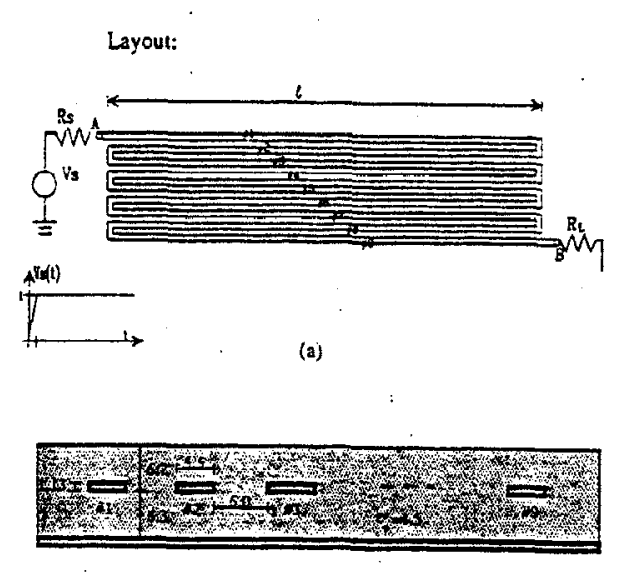

unit : mit. metat : Al

(b)

Fig.1 Serpentine delay line (a) tope view (b) cross sectional view.

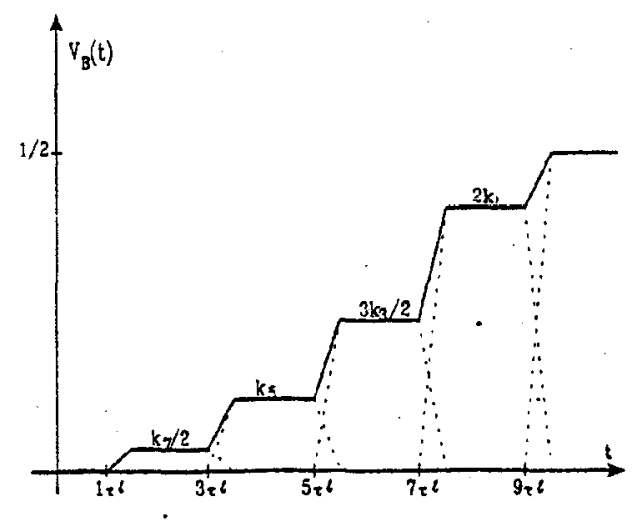

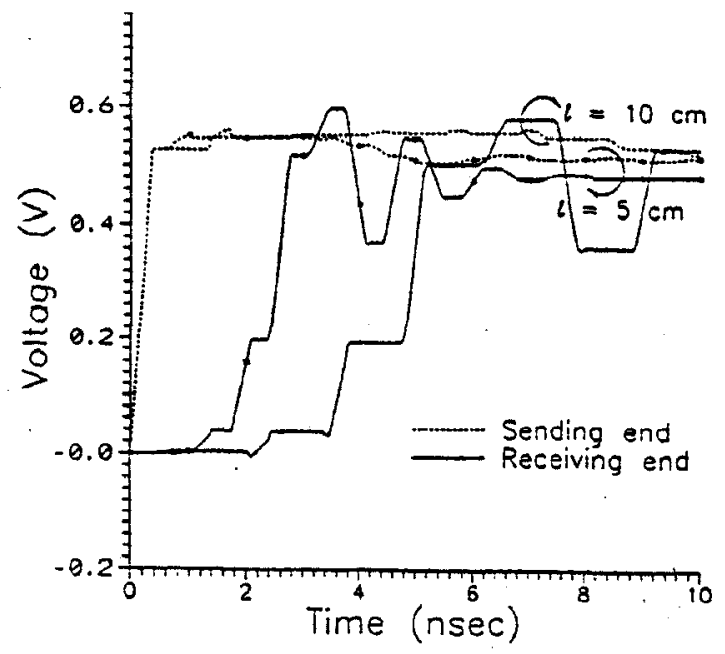

Fig.3 Simulated waveforms at sending and receiving ends.

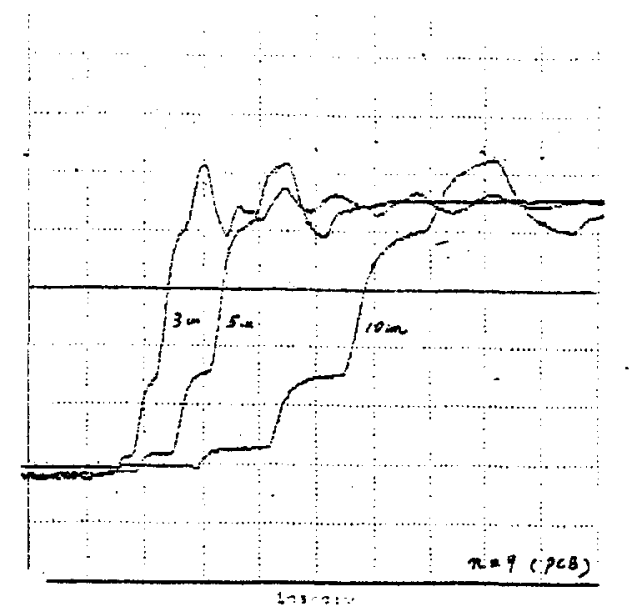

Fig.2 Receiving waveform by wave tracing analysis.
Fig.4 Measured waveforms at the receiving end. 